\title{
Analysis of Fungal Contamination on Commercially Sold Rice in Puerto Rico
}

\author{
Nicole Colón Carrión, Chad Lozada Troche \\ Department of Biology, University of Puerto Rico, Cayey, PR \\ Student: nicole.colon11@upr.edu \\ Mentor:chad.lozada@upr.edu
}

\begin{abstract}
Crops and stored grains are susceptible to pathogens that represent a threat to our health. The study presented herein compares the normal surface and endophytic fungal communities present on white and brown rice grains. One hundred grains of each rice variety was analyzed to determine their fungal contaminants and endophytes. Fungi were inoculated on SDA media, and purified in PDA media; morphological characterization was performed followed by amplification of the ITS region using PCR for all fungal isolates. Statistical analysis indicated significant differences between medium brown rice compared to white rice for surface and endophytic communities ( $\mathrm{p}$-value $\leq 0.05$ ). In addition, a higher fungal diversity was found on brown rice grains compared to white rice. This variation may be due to differences in the processing methods used for each rice grain type. BLAST analysis revealed the presence of toxigenic strains of Aspergillus flavus, A.oryzae, Penicillium verrucosum, and P. viridicatum. The study of fungal growth in rice grains can contribute to the minimization of mycotoxin production by its prevention and control; therefore, decreasing crop contamination and human exposure to their metabolites.
\end{abstract}

\section{KEYWORDS}

Fungi; Rice; Fungal Contaminants; Fungal Endophytes

\section{INTRODUCTION}

For years, rice has been one of the most important crops worldwide, not only for its nutritional value but also for the economic profit generated by its production and consumption. Produce and commercialized in approximately more than 25 countries, rice has become an essential nutrient in our daily diet. According to the Food and Agriculture Organization, in 2015 milled rice production reached approximately 491.5 million tons (milled eq.); with a feed use of 1.8 million tons (milled eq.). ${ }^{1}$ China, India, Indonesia, Bangladesh, Vietnam, and Thailand are described as the six major producers of this crop. Even though the United States production of rice is relatively low compared to the six top producers, it is still considered a major one. In 2015, U.S. rice exports increased 18\% from the prior year to a total of 3.5 million tons, the highest in 4 years; most of the yield was exported to Latin America. ${ }^{2}$ Puerto Rico imports $85 \%$ of their food products from the United States, including rice, based on the U.S. merchant marine acts, under the Foraker Act. This law states that entrance of every product into the island must be carried on U.S. shipments. ${ }^{3}$

Rice crops can be contaminated with mycotoxin-producing fungi. These pathogens are known to infest rice via two pathways: (1) in the field while rice is growing (field fungi); pre-harvest method, or (2) during the processing and storage after harvesting (storage fungi); post-harvest method. The presence or absence of mycotoxins depends on different factors such as genotype of the fungal isolate, interactions with other organisms on the substrate where they coexist, origin of the crop and its climatology, stress factors, available nutrition, physical damage due to insect activity, or agricultural and post-harvest practices. ${ }^{4,5}$ The major mycotoxins, based on the levels of toxicity, have been identified as aflatoxins, ochratoxins, fumonisins and trichothecenes. ${ }^{6}$ These can be produced by various genera including Aspergillus, Penicillium, Fusarium, Claviceps, Stachybotrys. ${ }^{6}$ Human exposure to mycotoxin has been linked to many neurological diseases, immunosuppression, carcinogenic effects, nephrotoxicity, and hepatotoxicity, among others. ${ }^{6-8}$

It is estimated that approximately $25 \%$ of the world cereal production is contaminated with mycotoxins or their derivatives. ${ }^{9}$ The study presented here is an initial effort to analyze endophytic and surface fungal contamination in milled white rice grains and brown rice grains commercially sold in Puerto Rico. Due to the level of importance, this crop possesses to human nutritional sustainability, the study of mycotoxin-producing fungi will provide insight for future prevention and control of exposure to mycotoxins, therefore decreasing global economic losses, crop contamination, and human exposure to fungal pathogens and their secondary metabolites. 


\section{METHODS AND PROCEDURES}

Collection of Rice Grains

Between 454 and 907 grams of milled rice samples were purchased from stores and supermarkets located in Caguas, Puerto Rico; standard packages sold for medium fortified brown rice contained less grain quantity (454 grams) when compared with medium fortified white rice (907 grams). Multiple batches were obtained at two-time points: August 2015 and June 2017, and analyzed one week after purchase; bags were stored at $25^{\circ} \mathrm{C}$, and ambient light. Once open, batches were not re-used or stored. Two different types of rice, medium fortified white and brown rice, were tested for surface and endophytic fungal isolates. Each group consisted of biological triplicates containing 50 grains per rice type. Both samples were selected from the same brand denominated as Brand A.

\section{Surface Fungal Isolation Assay}

Fungal Isolation was performed following Samson et al. (2002) protocol. ${ }^{10}$ A total of 150 grains per rice type were selected for analysis; $n=3,50$ samples per $n$. Grains were immersed in a solution of $0.4 \%$ of sodium hypochlorite for 2 min, rinsed with $\mathrm{dH}_{2} \mathrm{O}$, and dried for sterilization. Direct plating of grains was performed into Sabouraud Dextrose Agar (SDA) and incubated at $25^{\circ} \mathrm{C}$ for 7 days and ambient light; 5 particles per plate. Fungal colonies were sub-cultured and purified on Potato Dextrose Agar (PDA) under the same conditions.

\section{Endophyte Isolation Assay}

Fungal Isolation was carried out using a modification of the method described by Samson et al. (2002). ${ }^{10}$ One hundred fifty grains per rice type were selected for analysis; $n=3,50$ samples per $n$. Grains were half-cut and surface-sterilized with a solution of $0.4 \%$ of sodium hypochlorite for 2 min, rinsed with $\mathrm{dH}_{2} \mathrm{O}$ and dried. Plating of samples and purification of isolates was performed as described above.

\section{Control Assay}

Ten grains were surface-sterilized as described previously, dried, and autoclaved. Plating of samples and purification of isolates was performed as described above.

\section{Morphological Identification of Isolates}

Fungal characterization for surface and endophytic isolates was performed following Lozada-Troche et al. (1998) "Moist chambers" protocol. ${ }^{11}$ Chamber cultures consisted of Petri dishes containing a $75 \mathrm{~mm}$ microscopic slide placed above a V-shaped glass rod with a portion of SDA media over the slide and filled with $\mathrm{dH}_{2} \mathrm{O}$. Mycelia of isolates were transfer into the chamber's media and a cover with a glass slip. Chambers were maintained at room temperature and ambient light. After 96hrs, fungal structures were stained with lactophenol cotton blue to identify the genera according to their hypha, conidia and spore morphology. Isolates were identified following the Dugan (2006) identification guide. ${ }^{12}$

\section{$D N A$ Extraction}

Extraction and purification of DNA from fungal isolates were carried out using a modification of the protocol described by Liu et al. (2000). ${ }^{13}$ Hyphal tip transfer of samples was performed into SDA liquid media and incubated for 7 days at $25^{\circ} \mathrm{C}$ with a shaking speed of $40 \mathrm{rmp}$. Fungal isolates were centrifuged at $10,000 \mathrm{x} g$ for five minutes, and the supernatant discarded. Five hundred $\mu \mathrm{l}$ of lysis buffer was added to Eppendorf microtubes containing fungal hyphae and blended for ten minutes. Once homogenous, $150 \mu \mathrm{l}$ of $5 \mathrm{M}$ potassium acetate was added, vortexed, and centrifuged at $10,000 \mathrm{x} g$ for one minute. The supernatant was collected, $600 \mu \mathrm{l}$ of $100 \%$ isopropanol added, and tubes were spun at $10,000 \mathrm{x} g$ for two minutes. The resulting DNA pellet was washed using $500 \mu \mathrm{l}$ of $70 \%$ ethanol, then centrifuged, after which ethanol was removed, and residual ethanol was evaporated at $25^{\circ} \mathrm{C}$ for ten minutes. The pellet was re-suspended in $50 \mu \mathrm{l}$ of $10 \mathrm{Mm}$ Tris.

\section{Amplification of ITS region}

The Internal Transcribed Spacer (ITS) region one and two including 5.8S gene were amplified using specific primers ITS1 and ITS4. ${ }^{14}$ PCR amplifications were carried out in a total volume of $50 \mu$ containing $2.5 \mu \mathrm{M}$ of nucleotide mix, 20 pmol of each primer, 2.5 U of Taq Polymerase (Promega, Madison, USA), 1X reaction buffer and 20-50 ng of genomic DNA. Amplifications were performed in an Eppendorf Master Cycler ${ }^{\circledR}$ gradient (Brinkmann Instruments) using 30 cycles with the following parameters: 1 min denaturation at $94^{\circ} \mathrm{C}, 45 \mathrm{sec}$ annealing at $45^{\circ} \mathrm{C}, 1 \mathrm{~min}$ extension at $72^{\circ} \mathrm{C}$, and a final extension of 5 min at $72^{\circ} \mathrm{C}$. PCR amplifications were verified using a $1 \%$ agarose gel with UVIEW (Biorad) under UV light. Length of amplified fragments was determined by comparison with the migration of Lambda Hind III plus marker (Lambda Biotech Inc., St. Louis, USA). PCR amplicons were purified following the Zymoclean Gel DNA Recovery protocol. ${ }^{15}$ 
Sample Sequencing and Isolate Identification

The sense and reverse strands of the PCR products of the fungal strains were sequenced using the Applied Biosystems Big Dye ${ }^{\mathrm{TM}}$ Terminator v3.1 and ABI 3130 xl genetic analyzer (Applied Biosystems/ MDS SCIEX, Foster City, USA). DNA purified samples were sent to the Sequencing and Genomics facility, University of Puerto Rico-Río Piedras for sequencing. The DNA sequence data from fungal amplicons were analyzed with a BLASTn to identify the strains at the species level. ${ }^{16}$ Newly obtained sequences were deposited in GeneBank (Table 1).

\begin{tabular}{ll}
\hline Species & Accession numbers \\
\hline Aspergillus aculeatus & $\mathrm{KY} 006833, \mathrm{KY} 006835, \mathrm{KY} 006836, \mathrm{KY} 006838, \mathrm{KY} 006839$ \\
A. clavatus & $\mathrm{KY} 006827, \mathrm{KY} 006843, \mathrm{KY} 006845, \mathrm{KX} 944171, \mathrm{KX} 944177$ \\
A. flavus & $\mathrm{KY} 006825, \mathrm{KY} 006826, \mathrm{KY} 006828, \mathrm{KY} 006831, \mathrm{KY} 006832, \mathrm{KY} 006844$, \\
& $\mathrm{KX} 944169, \mathrm{KX} 944170, \mathrm{KX} 944180, \mathrm{KX} 944182$ \\
A. japonicus & $\mathrm{KY} 006834$ \\
A. oryzae & $\mathrm{KY} 006837, \mathrm{KY} 006846, \mathrm{KX} 944179$ \\
Fusarium equiseti & $\mathrm{KX} 944178$ \\
Penicillium citrinum & $\mathrm{KX} 944172, \mathrm{KX} 944174$ \\
P. polonicum & $\mathrm{KY} 006840, \mathrm{KY} 006841, \mathrm{KY} 006842$ \\
P. verrucosum & $\mathrm{KY} 006830, \mathrm{KX} 944181$ \\
P.viridicatum & $\mathrm{KY} 006829, \mathrm{KX} 944176$ \\
Penicillium sp. & KX944175 \\
Rinocladiella similis & \\
\hline
\end{tabular}

Table 1. New ITS sequences obtained in this study. Sequences were deposited in GeneBank; species name and accession number are listed.

\section{Statistical Analysis}

Colonization frequency (CF) was calculated as described by Suryanarayanan and Vijaykrishna (2001); stated in Equation 1. ${ }^{17}$ CF's were graph using Datagrap 4.1 (Visual Data Tools, Inc., 2006). Wilcoxon's test was performed to compare the difference in fungal communities between medium fortified white vs. brown rice for surface and endophytic isolates; $\mathrm{P} \leq 0.05$ was set to determine the significance of results. GraphPad contingency calculator (StataCorp LLC, TX) was used to determine P value.

$$
C F=\left(\frac{\text { number of rice grains colonized by a specific genera }}{\text { number of total rice grains analyzed }}\right) \times 100 \quad \text { Equation } 1 .
$$

\section{RESULTS}

A significant difference in surface and endophytic fungal communities between medium fortified brown vs. white rice Wilcoxon's test was conducted to determine a difference in fungal presence between medium fortified white vs. brown rice. A significant difference between medium brown rice compared to white rice was determined for surface and endophytic communities ( $\mathrm{p}$-value $\leq 0.05$ ) (Table 3). A number of fungal isolates obtain per replicate is presented in table 2 for each group. Control samples showed no fungal colonization.

\begin{tabular}{|c|c|c|c|c|}
\hline Mycoflora Type & Rice Type & N=1 & N=2 & N=3 \\
\hline Surface & White Rice & 5 & 4 & 2 \\
\hline & Brown Rice & 22 & 38 & 40 \\
\hline Endophytic & & & & \\
\hline & White Rice & 3 & 2 & 30 \\
\hline
\end{tabular}

Table 2. A number of fungal isolates obtain on rice grains. Each replicate consisted of 50 grains.

\begin{tabular}{|c|c|c|}
\hline Mycoflora Type & & P value \\
\hline Surface & White Rice vs. Brown Rice & 0.0495 \\
\hline Endophytic & White Rice vs. Brown Rice & 0.0463 \\
\hline
\end{tabular}

Table 3. P- value of medium fortified white $v$ s. brown rice for surface and endophytic communities. ( $\mathrm{n}=3$, 50 samples per replicate). 
Colonization frequency for surface communities

Identification of fungal isolates revealed strains of Aspergillus, Penicillium, Rhinocladiella, Mucor, and Fusarium. CF's were significantly higher for brown rice compared to white rice, with a higher frequency of Aspergillus strains (Figure 1). Rhinocladiella strains were only found on white rice, while strains of Fusarium and Mucor on brown rice. Furthermore, a significant amount of fungal isolates was identified as 'non-sporulators' due to their inability to develop a reproductive structure.

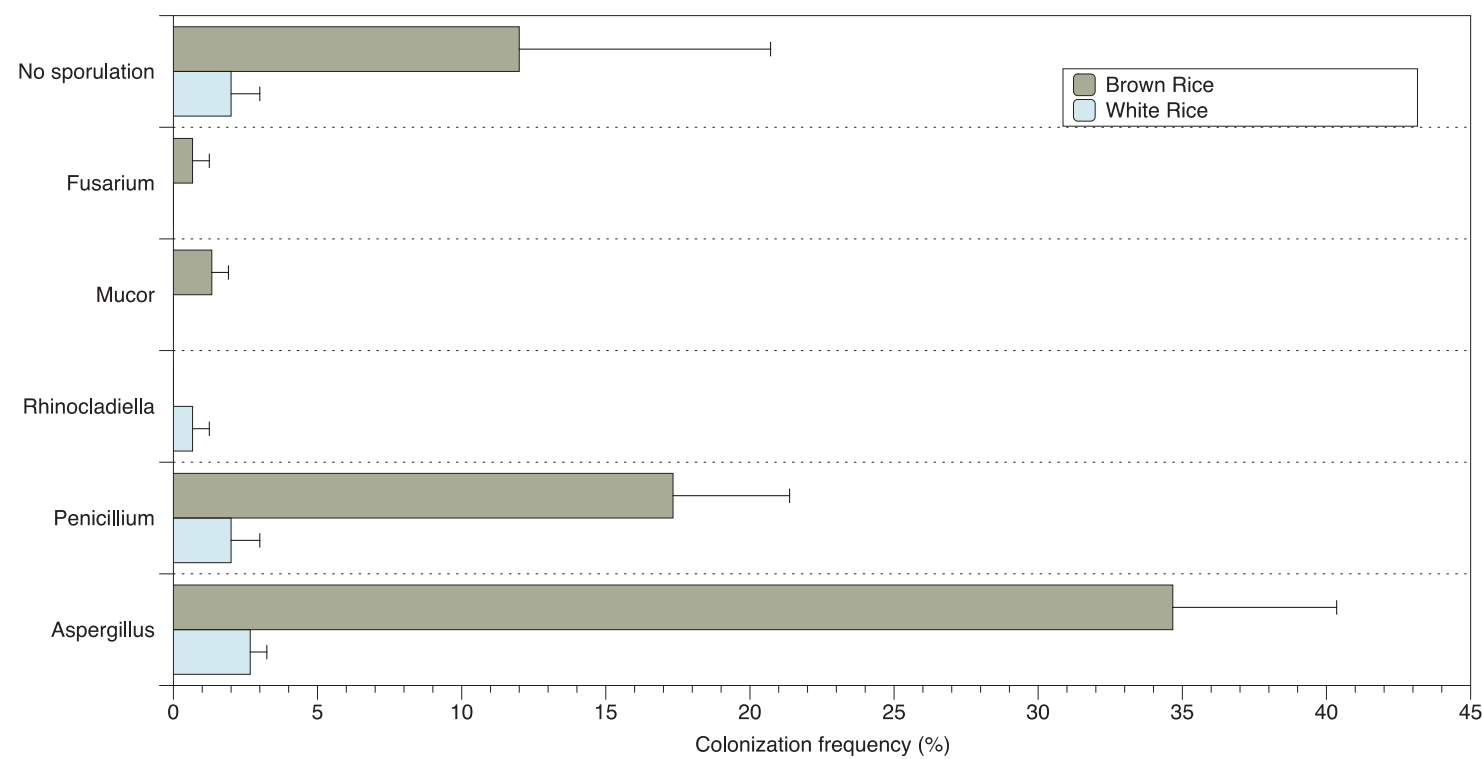

Figure 1. Colonization frequency for surface communities. \% age of CF's on medium fortified white and brown rice grains (three replicates per type).

\section{Blast Sequence Identification}

BLASTn sequence identification was only performed as $\mathrm{n}=1$. Results successfully identified strains of Aspergillus oryzae, Penicillium citrinum and Rinocladiella similis in medium fortified white rice. Strains of Aspergillus clavatus, Aspergillus flavus, Aspergillus oryzae, Fusarium equiseti, Mucor sp., Penicillium alli, Penicillium polonicum, Penicillium viridicatum, and Penicillium sp. were identified in medium brown rice. Endophytes consisted of strains of Aspergillus aculeatus, Aspergillus flavus, Aspergillus japonicas, and Penicillium verrucosum. Percentages of BLAST homology are presented in Table 4.

\begin{tabular}{|c|c|c|c|}
\hline Surface Communities & Rice Type & Endophytic Communities & Rice Type \\
\hline Aspergillus clavatus (99\%) & B & Aspergillus aculeatus (98\%) & W, B \\
\hline Aspergillus flavus (99\%) & B & Aspergillus flavus (96\%) & Aspergillus japonicus (99\%) \\
\hline Aspergillus oryzae (100\%) & W, B & Penicillium verrucosum (99\%) & B \\
\hline Fusarium equiseti (98\%) & B & & \\
\hline Mucor sp. & B & & \\
\hline Penicillium alli (98\%) & B & & \\
\hline Penicillium citrinum (99\%) & W & & \\
\hline Penicillium polonicum $(97 \%)$ & B & & \\
\hline Penicillium viridicatum $(98 \%)$ & B & & \\
\hline Penicillium sp. $(99 \%)$ & W & & \\
\hline Rinocladiella similis (98\%) & & & \\
\hline
\end{tabular}

Table 4. Fungal isolates in surface and endophytes mycoflora obtain in this study. Percentage indicates BLAST homology. W $=$ white medium fortified rice, $\mathrm{B}=$ brown medium rice. ( $\mathrm{n}=1$, three replicates per group). 


\section{DISCUSSION}

Statistical analysis indicated significant differences between medium brown rice compared to white rice for surface and endophytic communities ( $\mathrm{p}$-value $\leq 0.05$ ); suggesting that a higher proportion of medium brown rice contains fungal contaminants. Microscopic fungal identification indicated the presence of genera such as Aspergillus, Penicillium, Rhinocladiella, Mucor, and Fusarium, with CF's ranging from 2\% to 34\% (Figure 1). Furthermore, a higher fungal diversity was observed on brown compared to white rice grains. BLAST sequence identification showed the presence of putative mycotoxin-producing fungi Penicillium citrium in white medium fortified rice, and Aspergillus flavus and Penicillium viridicatum in brown medium fortified rice. The high levels of fungal growth in medium brown rice compared to white rice may be due to harvesting and milling methods. After harvesting, grains undergo a drying and hulling process, at this point, no further processing is necessary for brown rice. However, white rice is subjected to a milling and enriching process which ensures the removal of the outer bran layer and provides the white gloss aspect. ${ }^{18}$ The removal of this layer leads to a loss in protein, vitamins, minerals and lipids levels; utilized by fungi during their nutrition. This may implement a significant reduction of mold's nutrients leading to a reduction in fungal growth and diversity on white rice.

In studies conducted with rice in Cuba and sorghum in Argentina, other researchers found the presence of filamentous and potential mycotoxin-producing genera Aspergillus, Fusarium, and Penicillium, as well as phytopathogenic genera Bipolaris, Curvularia, Alternaria, Pycularia, and Cercospora in rice (Almaguer and Rodriguez-Rajo, 2012), and Alternaria, Aspergillus, Cladosporium, Curvularia, Fusarium, Penicillium, and Phoma in sorghum (Gonzalez et al., 1997). 19, 20 Furthermore, studies conducted with rice in Vietnam revealed the presence of three main genera: Aspergillus, Fusarium, and Penicillium; with focus on toxigenic strains Aspergillus flavus, Aspergillus ochraceus and Penicillium citrinum. ${ }^{21}$ In southern regions of the United States, previous reports showed that Aspergillus flavus and Fusarium verillicoides represent high contaminants for corn samples, while Fusarium species are most common in rice; nevertheless, rice seems to exhibit more resistance to fungal contamination and mycotoxin production than other crops. ${ }^{22}$ Similarly to these studies, our results showed the presence of Aspergillus, Fusarium, and Penicillium genera's in medium fortified white and brown rice; while species analysis revealed the presence of Aspergillus flavus and Penicillium citrinum.

The high occurrence of fungal contamination in commercially sold rice grain in Puerto Rico may be due to poor post-harvesting methods. Extrinsic factors such as humidity, water content, and temperature are a vital factor for fungal infection. Water content is the principal environmental factor that influences the three stages of germination, particularly the first stage: swelling of the spore. ${ }^{23}$ Furthermore, temperatures ranging from $15-40^{\circ} \mathrm{C}$ promote fungal infection. ${ }^{23}$ When imported, grains are prone to constant temperature and humidity changes which contribute to fungal proliferation. Because of the great importance this crop possesses, it is vital that strict pre-harvest and post-harvest methods be applied in order to prevent and reduce the incidence of fungal contamination, and possible mycotoxin production. Thus, the measures applied to reduce and minimize contamination should focus towards the prevention against fungal development in the field, as well as the production of secondary metabolites. ${ }^{4}$

\section{CONCLUSION}

The present study represents an initial effort to reveal the occurrence of surface and endophytic fungal microflora contamination in medium fortified white and brown rice commercially sold in Puerto Rico. The results have shown a higher occurrence of fungal contamination in brown rice grains compared to white rice for surface and endophytic communities. Nevertheless, furthers studies with a higher number of replicates, as well as consideration of other rice brands need to be conducted to establish a specific pattern. Taxonomic results showed a higher fungal diversity on brown rice compared to white rice. In addition, potential mycotoxin-producing species were identified in both white and brown rice grains; Penicillium citrium in white medium fortified rice, and Aspergillus flavus and P. viridicatum in brown medium rice. Further studies should focus on identification, quantification, and prevention of mycotoxins in rice grains. The measures applied to reduce and minimize mycotoxins should focus towards two different aims: the prevention against contamination and fungal development in the cereal, as well as the production of secondary metabolites; and the detoxification of mycotoxins when they occur in the grain. ${ }^{4}$ For the latest, decontamination of mycotoxin and mycotoxin-producers by cooking methods had been successfully implemented. Palavaras et al. (2004) showed a 72-89\% reduction of mycotoxin levels in rice grains after different cooking methods. ${ }^{4,24}$ Hence, autoclaving practices will implement a great and accessible post-harvest method for the reduction of mycotoxin-producers and their metabolites in food samples in industrial settings; as shown in our previous control assay (refer to method section).

\section{ACKNOWLEDGMENTS}

Authors thank Dr. Carlos Betancourt-Lopez for reviewing the manuscript. Authors are also thankful for financial assistance provided by FIDI (Fondo Institucional para el Desarrollo de la Investigación) at the University of Puerto Rico - Cayey, and the Puerto Rico Louis Stokes Alliance for Minority Participation (Grant Number: 1400868). This project was made possible with support from the Sequencing and Genomics Facility of the UPR Río Piedras \& MSRC/UPR, funded by NIH/NIGMS-Award Number P20GM103475. 


\section{REFERENCES}

1. Food and Agriculture Organization, FAO. Rice Market Monitor 2015, http.// wmw.fao.org/ economic.RMM (accessed Feb 2016)

2. United States Department of Agriculture Foreign Agricultural Service, U.S.D.A. Rice Outlook 2016, http:/ / www.fas.usda.gov/data/grain-world-markets-and-trade (accessed Jan 2016)

3. Bender, L. D. (1998) Economics and business, in The American presence in Puerto Rico 1st ed., 71-77, Publicaciones Puertorriquenas, Puerto Rico.

4. Ferre, F. S. (2016) Worldwide occurrence of mycotoxins in rice. Food Control 62, 291-298. https:// doi.org/10.1016/j.foodcont.2015.10.051

5. Bhat, R., Rai, R. V., \& Karim, A. A. (2010) Mycotoxins in Food and Feed: Present Status and Future Concerns, Compr Rev Food Sci Food Saf 9, 57-81.

6. Serrano, H. A., \& Cardona, N. C. (2015) Micotoxicosis y micotoxinas: generalidades y aspectos básicos, Rev CES Med 29, 143152.

7. Marroquín, A. G., Johnson, N. M., Phillips, T. D., \& Hayes, A. W. (2014) Mycotoxins in a changing global environment - A review. Food Chem Toxicol 69, 220-230. https:// doi.org/10.1016/j.fct.2014.04.025

8. Gimeno, A., \& Martins, M. L. (2011) Mycotoxins and mycotoxicoses more significant in animals and humans, in Mycotoxins and mycotoxicoses in animals and humans 3rd ed., 65-81, Special Nutrients, Florida.

9. Aydin, A., Aksu, H., \& Gunsen, U. (2011) Mycotoxin levels and incidence of mould in Turkish rice, Environ Monit Assess 178, 271-280. https:/ / doi.org/10.1007/s10661-010-1688-9

10. Samson, R. A., Hoekstra E. S., \& Frisvad J. C. (2004) Introduction to food- and airborne fungi 7 th ed., Centraalbureau voor Schimmelcultures, Utrecht.

11. Lozada-Troche C., Ortiz, B., \& Betancourt, C. (1998) Moist chambers, in "Introducción a la Micología Médica. Imprenta Universitaria” 1st ed., 16, Recinto Universitario de Mayagüez, Puerto Rico.

12. Dugan, F. M. (2006) The identification of fungi: an illustrated introduction with keys, glossary, and guide to literature, APS, St. Paul, Min.

13. Liu, D., Coloe, S., Baird, R., \& Pendersen, J. (2000) Rapid mini-preparation of Fungal DNA for PCR, J Clin Microbiol $38,471$.

14. White, T. J., Bruns, T., Lee, S., \& Taylor, J. (1990) Amplification and direct sequencing of fungal ribosomal RNA genes for phylogenetics, in PCR Protocols: a Guide to Methods and Applications (Innis, M. A., Gelfand, D. H., Sninsky, J.J., White, T., Ed.) 1st ed., Academic Press, New York.

15. ZYMO RESEARCH CORP. Zymoclean ${ }^{\mathrm{TM}}$ Gel DNA Recovery Kit Instruction Manual, http:/ / www.zymoresearch.com/downloads/dl/file/id/34/d400li/pdf (accessed Dec 2015)

16. Altschul, S.F., Gish, W., Miller, W., Myers, E. W., \& Lipman D.J. (1990) Basic local alignment search tool, J Mol Biol 215, 403410.

17. Suryanarayanan, T.S., \& Vijaykrishna, D. (2001) Fungal endophytes of aerial roots of Ficus benghalensis, Fungal Divers 8, $155-161$.

18. Yadav, B. K., \& Jindal, V. K. (2001) Monitoring milling quality of rice by image analysis, Comput Electron Agric 33, 19-33.

19. Almaguer, M., Rojas, T. I., \& Rodríguez-rajo, F. J. (2012) Airborne fungal succession in a rice field of Cuba, Eur J Plant Pathol 133, 473-482. https:/ / doi.org/10.1007/s10658-011-9921-0

20. Mart, E. J., \& Resnik, S. L. (1997) Fungi associated with sorghum grain from Argentina, Mycopathologia 139, 35-41.

21. Trung, T. S., Bailly, J. D., Querin, A., Le Bars, P., \& Guerre, P. (2001). Fungal contamination of rice from South Vietnam, mycotoxinogenesis of selected strains and residues in rice. Revue de Médecine Vétérinaire, 152(7), 555-560.

22. Abbas, H. K., \& Shier, W. T. (2009). Mycotoxin contamination of agricultural products in the southern United States and approaches to reducing it from pre-harvest to final food products. Mycotoxin prevention and control in agriculture. W ashington, DC: American Chemical Society, 37-58.

23. Gottlieb, D. (1950) The physiology of spore germination in fungi, Bot Rev 16, 229-257.

24. Palavras, C., Simionato, E., Sylos, M. R. S., and De, C. M. (2004). Effect of cooking on the levels of AFB1 and ochratoxin A in rice. Brazilian J. Food Technol. 7(2):167-171.

\section{ABOUT THE STUDENT AUTHOR}

Nicole Colón Carrión graduated in June 2016 from the University of Puerto Rico Cayey Campus with a bachelors degree in Biology. She has been working along with Dr. Lozada in this research project for two years; where she was awarded two research fellowship by the Puerto Rico Louis Stokes Alliance for Minority Participation Program, and one by the American Society for Microbiology Undergraduate Research Capstone Program. Furthermore, she had the privilege to present in a series of regional and national meetings, in which she was awarded Second Place Oral Presentation by the Puerto Rico Society for Mycologists. After graduation, she worked as a postbaccalaureate student at Michigan State University in the Department of Pharmacology and Toxicology studying the role of AMPA receptors in methylmercury-induced toxicity in hiPSC-MN. She is currently pursuing her graduate studies at the University of Arizona in the Arizona Biological and Biomedical Sciences Program specializing in Plant Pathology, where she was currently awarded an NSF-GRFP fellowship. 


\section{PRESS SUMMARY}

For years, rice has been one of the most important crops in the world. Approximately $75 \%$ of the human population includes it in their daily diet because of its nutritional value. However, these crops can be attacked by various pathogens, leading to production losses, and adverse health effects in animals and humans. One of the most important groups of pathogens and major crop contaminants are fungi. The study presented herein compares the surface and endophytic fungal microflora present on medium fortified white and brown rice commercially sold in Puerto Rico. The goal of this research is to identify whether commercially sold rice in Puerto Rico are contaminated with fungal pathogens, and the difference in fungal incidence between medium fortified white vs. brown rice The identification and classification of these organisms is vital to elucidate their behavior and mechanisms of action in the environment, as well as designing new methods for prevention and control; therefore, decreasing crop contamination and human exposure. 